\title{
IMPLEMENTATION OF WEB RESOURCE SERVICE TO PRODUCT DESIGN
}

\author{
Baoli Dong ${ }^{1,2}$, Sumei Liu ${ }^{1}$ \\ ${ }^{\prime}$ Department of Mechnical Engieering, Taiyuan University of Technology and Science, China; \\ Email: tydbl@hotmail.com; ${ }^{2}$ Department of Mechnical Engieering, Zhejiang Univ., China.
}

\begin{abstract}
Manufacturing enterprises needs the wider external resources during the product design phrase. A typical web resource service approach is presented in this paper. It realizes Web-based manufacturing resource discovery, process and application in product design. This pattern addresses on the differences of service ways considering different types of resources. The relevant enabling technologies are put forward. Manufacturing resource information is achieved through Web search engine and processed with semantic Web. Meanwhile, the Web resource repositories such as Web parts library and distributed knowledge bases are set up preliminarily. Finally, the two application cases of Internet-based collaborative product design are introduced briefly.
\end{abstract}

Key words: $\quad$ Manufacturing Service, Web Resource, Product Design.

\section{INTRODUCTION}

Manufacturing enterprises must respond speedily to the market and customer needs ${ }^{1,2}$. The increasing product complexity and the globalization manufacturing further necessitate a wide-ranging distributed manufacturing resource demand involved in the product development stage. Product development more and more requires that manufacturing enterprises possess of the higher design level, advanced manufacturing equipments, better network communication environment ${ }^{3}$. However, the distribution of manufacturing resources is unbalanced. Most enterprises are incapable of satisfying these conditions and unable to implement product development alone. As a possible solution, the Web sharing of qualified manufacturing resources can help them utilize the external resources adequately. For this

This project is supported by the Key Project of Ministry of Education of China under the grant No.211CERS12.

Please use the following format when citing this chapter:

Dong, Baoli, Liu, Sumei, 2006, in International Federation for Information Processing (IFIP), Volume 207, Knowledge Enterprise: Intelligent Strategies In Product Design, Manufacturing, and Management, eds. K. Wang, Kovacs G., Wozny M., Fang M., (Boston: Springer), pp. 972-977. 
purpose, the enabling technologies of Web-oriented manufacturing resource discovery, integration and sharing services for product design are analyzed in the following sections.

\section{RESOURCE SERVICE AND KEY TECHNOLOGIES}

Product development is viewed as integration and sharing process of design and manufacturing resources ${ }^{4}$. Manufacturing resources available on the Web are much more extensive and the application ways are of diversity. Manufacturing resources can be divided into hardware, software, data and knowledge 5 . The unified data exchange standards like STEP, XML and semantic description like RDF, Ontology realize the in-depth data sharing and semantic services. The interlinking and interoperability through Internet directly for most software and hardware resources are difficult at present due to the technical and management factors. So Web resource services can help the enterprises to get external service resources and implement collaborative design and manufacturing. Considering the actual enterprise needs and product design process, we address to the following key technologies relating to Web manufacturing resource services.

- Web resource discovery: The enterprises need to acquire the external existing resources timely, apply them to enterprise decision-making and design activities fully. So resource discovery affects the succeeding resource integration and sharing process. We employ Web search, information processing and data mining applied to enterprise intelligence collection, knowledge management and decision analysis relating to product design activities. The Web manufacturing information and knowledge are achieved through focus crawling, information extraction and data mining, thus provide service contents such as resource navigation to enterprises.

- Web resource repository: Shared manufacturing resources determine the service quantity, range and quality. Web resource repositories realize resource integration, sharing and reuse. They can be divided into two types: one is information base and resource portal through automatic search from the Web, the other is that enterprises issue and provide such resource as distributed parts bases and knowledge ones. Both provide the general resource retrieval services, parts downloading and knowledge management.

- Web sharing supportive environment: Web resource applications need a communication, coordination and cooperation network supportive environment. Considering resource difference, the sharing tools consist of network conference, white board, RPC and etc, which conduce to cooperative product development among users. 


\section{APPLICATION CASE}

\subsection{Manufacturing information and resource discovery}

The enterprise portals and domain sites contain abundant of Web manufacturing resources and information. The general search engines like Google and Baidu can't satisfy the search requirement of enterprise resources and the precision ratio is very low. The active and open Web resource discovery aiming at manufacturing field is an effective approach to search specialized resources. It can extend the acquiring range of shared resources and improve the search accuracy. Fig. 1 specifies the overall process of Web resource discovery.

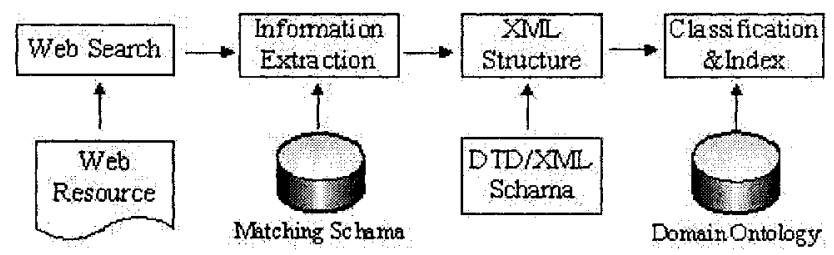

Figure 1. Web discovery and semantic transaction of manufacturing resource

Focus crawling can guarantee the search accuracy to specialized resources on the $\mathrm{Web}^{6}$. To a certain type of topic enterprise site, website topic feature modeling is a critical issue for focus crawling. The content and structure characteristics of different topic sites are obvious distinct. The semantic of feature text reflect its topic. The semantic text information extracted from the site is mapped to HVSM-based topic feature vector ${ }^{7}$. Then the topic classifier using support vector machine algorithm (SVM) determines its topic. The search engine only rambles topic-specific sites and gathers relevant Web pages.

The wrapper deals with parsing page contents and extracting manufacturing information. Web pages are of semi-structured documents, and manufacturing information are mixed with HTML tags and other useless contents. They are normalized the well-structured ones firstly, and converted to DOM (Document Object Tree) tree with DOM API of W3C. DOM tree determines the locating tags and extraction path of HTML document ${ }^{8}$. Considering the existing structure forms of HTML documents are very complicated, the extraction models are achieved by the Extraction Rule Learning Algorithm (ERLA) ${ }^{8}$. According to the definite pattern matching rules, the manufacturing information is extracted through the prefix and suffix forms of table attribute terms like product name, specification and price, etc. The extraction models include the locating tags, keywords, etc. 
Finally, the finely granular information is extracted and structured through these processes above mentioned, and saved to the resource database of server. Therefore, the service system can gather specialized resource information automatically and update timely.

To enhance the accuracy of resource retrieval, Resource Description Framework (RDF) is employed to describe, categorize and index extracted information. As a unified metadata description norm based on XML syntax, RDF strengthens the semantic retrieval ability ${ }^{9}$. It is independent of specific fields, so RDF Schema (RDFS) defines the domain taxonomy of resource description, and constructs the hierarchy model of manufacturing resource entity types such as enterprise, service, product and function. This strengths the resource description capacity of RDF and provides the semantic support for resource in-depth application. For example, the service information about mould design that Institute A provides, whose URL address is "http://www.amtcn.org/", can be described with RDF/XML as following.

$$
\begin{aligned}
& \text { < ? Xml version="1.0" encoding="UTF-8" ? > } \\
& \text { < rdf:RDF xmlns:rdf="http://www.w3.org/1999-02-22-rdf-syntax-ns\#" } \\
& \text { xmlns:s="http://description.org/schema/" > } \\
& \text { < rdf:Description about=" http://www.amtcn.org/" > } \\
& \text { < rdf:type rdf:resource=" http:// description.org / term\#Service"/ > } \\
& \text { < s:Item > automobile mould </s:Item > } \\
& \text { < s:Creator > Institute A</s:Creator > } \\
& \text { </rdf:Description > } \\
& \text { </rdf:RDF > }
\end{aligned}
$$$$
\text { < rdf:type rdf:resource }=" \text { http:// description.org / term\#Service"/ > }
$$

The extracted resource metadata information is processed with $\mathrm{RDF} / \mathrm{XML}$. While the user send the resource search request, the server parses the RDF-based resource documents with Jena, converts retrievalmatching results to XML form and offers the semantic search service. The prototype system is developed with Java. The search objects aim at the manufacturing equipments, software, products and suppliers, etc. Fig. 2 shows a resource discovery example. The users can get various enterprise and service information results related to querying contents.

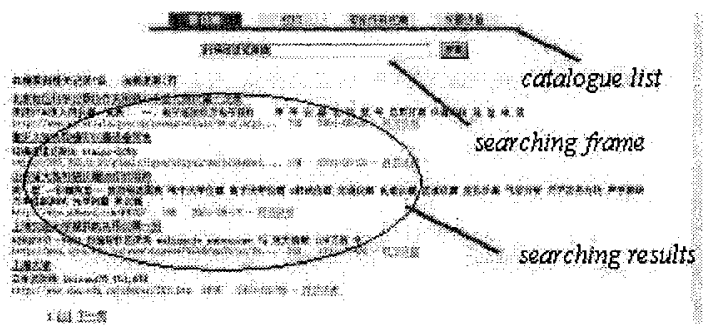

Figure 2. Resource search results of mould enterprises and organizations 


\subsection{Web parts library}

Web parts library is one of design resource bases for rapid product development. The enterprise is able to issue its product models and technical files to Web parts library. Other enterprises lacking design capacities can retrieval, preview, download and reuse the needed product models from it directly. The system architecture of Web parts library is shown as Fig.3. The service information contain the parts geometric models and other relevant contents relating to parts like supplier information.

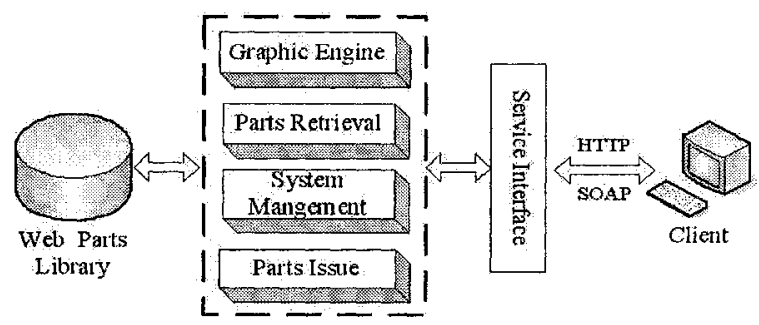

Figure 3. Architecture of Web parts library

As to parts retrieval, the modeling of parts family adopts the international standard ISO13584. This standard defines the product data representation and exchange format ${ }^{10}$. Parts description information includes two parts: parts data dictionary and its concrete contents. The former defines the parts type and relevant attributes, provides the parts classification identification standard. The latter is represented with XML syntax according to the parts classification standard and attribute elements by the data dictionary. The enterprise uploads the parts models by ISO13584 to Web parts library. The users retrieval parts information in terms of parts family and parts function characteristics. Fig. 4 shows that the users can retrieval, browse and download parts information of tire mould through Web parts library.

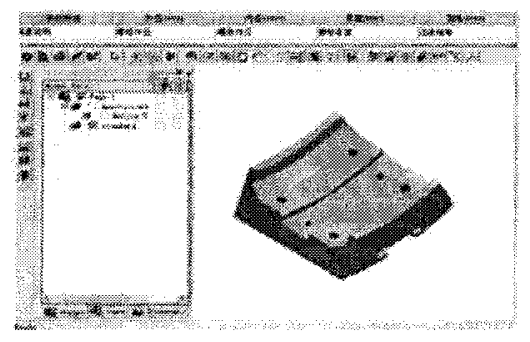

Figure 4. Browsing and downloading service of parts model 
Web parts library provides a successful sharing environment and a seamless interactive channel through Internet. It integrates with the existing commercial design system and enterprise operational activities, allows distributed users to exchange and share product visualized information and meets collaborative work requirements.

\section{CONCLUSION}

In this paper, the Web-oriented resource discovery and sharing service pattern is introduced, and the key implementation technologies are discussed in detail. These service ways meets the enterprise demand on quality specialized resources from the Web. We have built a prototype system towards the in-depth and overall sharing of network manufacturing resources for production development. Relevant services realize resource discovery and reuse in the whole product development cycle. Up till now, they have applied to the diversified application service objects for various product development objects covering mould, automobile parts, pump, clothing, medicine, etc. This resource-sharing service method has certain of instruction signification for rapid collaborative product development of manufacturing enterprises.

\section{REFERENCES}

1. Xie, S., Huang, H., Tu, Y., (2002), A WWW-based information management system for rapid and integrated mould product development, Advanced Manufacturing Technology 20(1), 50-57.

2. Huang, G., (2002), Web-based support for collaborative product design review, Computers in Industry 48(1), 71-88.

3. Huang, G., Lee, S. and Mak, K. L., (2001), Synchronized web applications for product development in the 21st century, Advanced Manufacturing Technology 18(8), 605-613.

4. Zhou, S., Chin, K., Xie, Y., et al, (2003), Internet-based distributive knowledge integrated system for product design, Computer in Industry 50(2), 195-205.

5. Yang, T., Wang, Y., Xiao, T., et al, (2003), Resource discovery in context of Internet-based manufacturing, Computer integrated Manufacturing System 9(1), 47-51.

6. Chakrabarti, S., Dom, B., Berg, M., (1999), Focused crawling: a new approach to topic specific web resource discovery, Computer Networks 31(11), 1623-1640.

7. Dong, B., Qi, G., Gu, X., (2005), Domain-specific website recognition using hybrid vector space model, Lecture Notes of Computer Science 3739, 840-845.

8. Li, X., Gu, Y., (2002), DOM-based information extraction for the web sources, Chinese Journal of Computers 25(2), 526-533.

9. Cai, M., Lin, L., Chen, G., et al, (2003), Intelligent resource discovery in Internet-based manufacturing, Computer Integrated Manufacturing System 9(7), 589-594.

10. Yang, Z., Qi, G., Gu, X., (2005), Research on web parts library for mass customization, Computer Integrated Manufacturing System 11(4), 541-547. 